\title{
Comparing confocal immunofluorescence microscopy (CIM) vs. freeze fracture replica immunogold labeling (FRIL) for cell-specific localization of plasma membrane proteins in the mammalian CNS.
}

\author{
T. Yasumura*, K.G.V. Davidson*, C.S. Furman*, J.I. Nagy** and J.E. Rash* \\ *Department Biomedical Sciences, Colorado State University, Fort Collins, CO 80523 and \\ **Department of Physiology, University of Manitoba, Winnipeg, Manitoba Canada, R3E 3J7
}

In the past decade, light microscopy has undergone a resurgence based on the use of confocal microscopy and immunofluorescence labeling, particularly the localization and three-dimensional mapping of membrane proteins. For example, connexins have been mapped to gap junctions in liver hepatocytes, where cells are essentially cuboidal, and identification of the cells forming a particular gap junction is not at issue. However, in the mammalian CNS, where most neuronal and glial processes are smaller than the limit of resolution of light microscopy, and where there are many cell types and possible combinations of coupling partners, assignment of connexin proteins to individual cell types is almost never possible by confocal microscopy. Localization of a specific membrane protein to a specific cell or cell pair by CIM faces three major difficulties: 1) With a maximum of three or four fluorphores available for use at one time, it is not possible to use fluorescent markers simultaneously to identify the four major cell types, and in addition, to label connexins/gap junctions. As a consequence, most investigators have used only one of the available fluorescent markers to visualize a single cell type (e.g., neurons) and one or two fluorophores to visualize target connexins. With other cells and cell processes not labeled or visualized, the images often have been erroneously interpreted as demonstrating the fluorescent connexin within the plasma membrane of the fluorescently-visualized cells. 2) The limit of resolution of confocal microscopy $(0.2 \mu \mathrm{m}$ in blue wavelengths and $0.4 \mu \mathrm{m}$ in red wavelengths, and double those values in the Z-axis) is greater than the diameter of most cell processes in the CNS. This means that three or more cell processes and six or more plasma membranes are often present in the smallest volume resolvable by CIM (Fig. 1).

In contrast, FRIL has several distinct advantages over CIM: A) In most instances, cells are positively identified based on ultrastructural features, reserving immunogold labels for identification of membrane proteins. B) With the development of immunogold labels of at least five discrete size classes (e.g., $5 \mathrm{~nm}, 10 \mathrm{~nm}, 15 \mathrm{~nm}, 22 \mathrm{~nm}$ and $30 \mathrm{~nm}$ ), as many as five or more membrane proteins may be localized to ultrastructurally-identified cells. C) In addition, FRIL has the distinct advantage of direct visualization and unambiguous identification of ultrastructurally-defined gap junctions in ultrastructurally-identified cell processes. D) The ability to visualize and quantify connexins and to quantify immunogold labels by FRIL allows us to answer questions not addressable by CIM, including determination of the relative labeling efficiency of immunogold labels vs. fluorescent labels for each class of connexins present in individual gap junctions (Fig. 2). E) Because each immunogold bead represents a separate labeling event, and because the number of labeled gap junctions and immunogold beads for each connexin may number in the hundreds and tens of thousands, respectively, one may use FRIL to ascertain the relative amount of a particular connexin in gap junctions of a particular cell class. For example, we have labeled more than 5000 astrocyte gap junctions with more than 100,000 gold beads for $\mathrm{Cx} 43$, with no $\mathrm{Cx} 43$ detected in gap junctions of neurons or oligodendrocytes. Thus, we conclude that if $\mathrm{Cx} 43$ is present in neuronal gap junctions, it is present at a density at least five orders of magnitude lower than in astrocytic gap 
junctions. Similarly, if $\mathrm{Cx} 32$ is present in neuronal gap junctions, it is present at a density at least three orders of magnitude lower than in oligodendrocyte gap junctions. We conclude that of the six CNS connexins tested to date, $\mathrm{C} \times 36$ is present in most neuronal gap junctions, and that $\mathrm{Cx} 26, \mathrm{Cx} 29$, $\mathrm{Cx} 30, \mathrm{Cx} 32$, and $\mathrm{Cx} 43$ are not present in neuronal gap junctions. Thus, we have used FRIL to identify the connexins present in gap junctions of each cell type, and then redesigned our CIM experiments to confirm that each connexin is obligately associated with the appropriate cell type.

\section{References:}

1. Fujimoto, K., J. Cell Sci. (1995) 3443.

2. T. Yasumura et al., Microsc. Microanal. (Suppl. 2) (1997) 345.

3. J.E. Rash et al., Cell Tissue Res. (1999) 307.

4. J.E. Rash et al., J. Neurosci. (2001) 1983.

5. This research was supported by NIH NS38121 and NS39040.
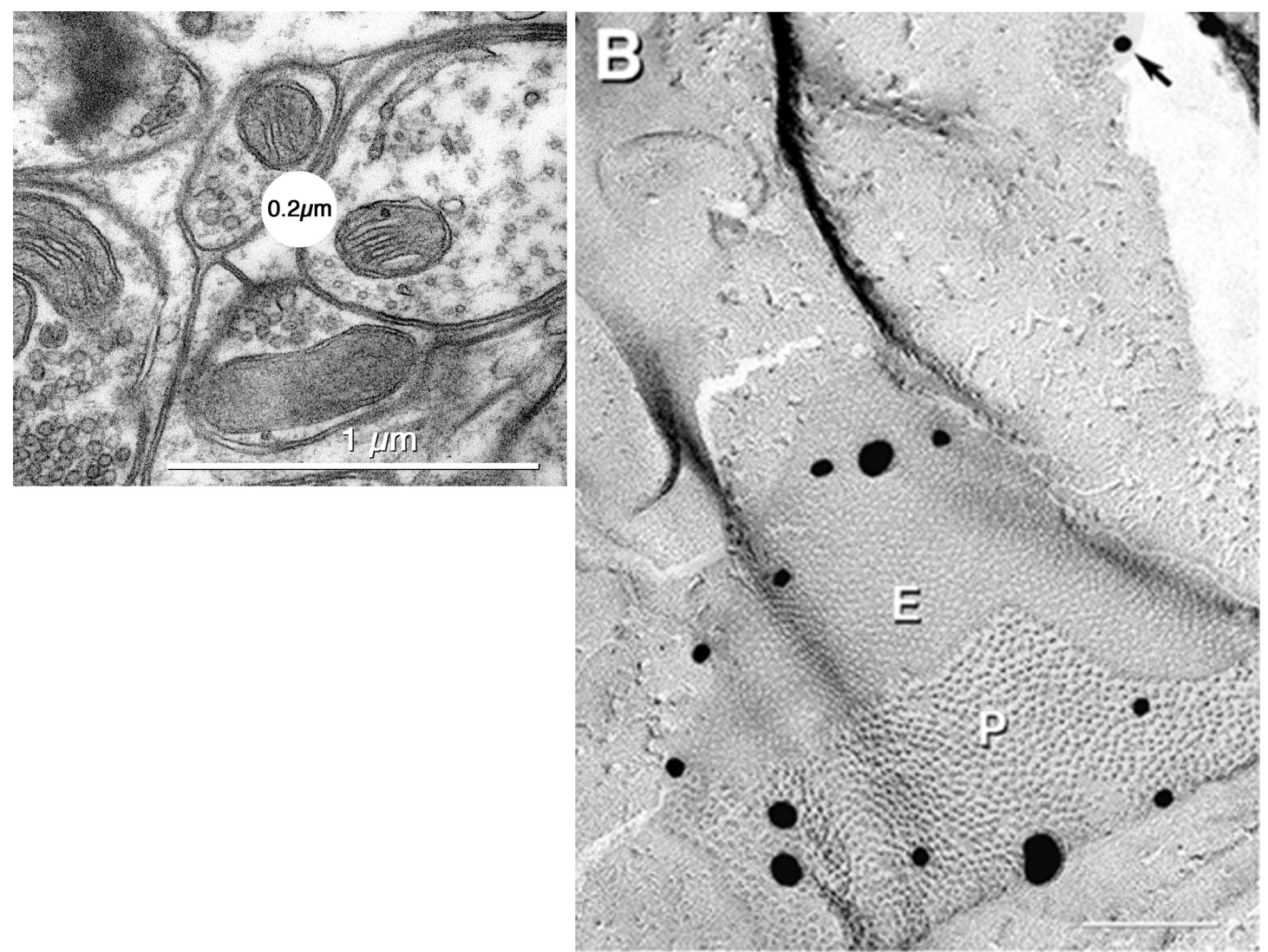

\section{B. $\mathrm{Cx} 43=20 \mathrm{~nm}$ gold $\mathrm{C} \times 30=30-40 \mathrm{~nm}$ gold $=10 \mathrm{~nm}$ gold (none present)}

Figure 1. Limit of resolution of confocal microscopy in blue wavelength $(0.2 \mu \mathrm{m})$ superimposed on thin section image from adult rat spinal cord.

Fig 2. (B) Triple-immunogold labeling of astrocyte gap junction. Cx30 (30-40nm gold) and Cx43 (20nm gold) are present, but Cx32 (10nm gold) is not present. 